\title{
Prediction of Short-Term Cardiovascular Events Using Quantification of Global Myocardial Flow Reserve in Patients Referred for Clinical ${ }^{82}$ Rb PET Perfusion Imaging
}

\author{
Kenji Fukushima ${ }^{1}$, Mehrbod S. Javadi ${ }^{1}$, Takahiro Higuchi ${ }^{1}$, Riikka Lautamäki ${ }^{1}$, Jennifer Merrill ${ }^{1}$, Stephan G. Nekolla ${ }^{2}$, \\ and Frank M. Bengel ${ }^{1,3}$ \\ ${ }^{I}$ Division of Nuclear Medicine, Russell H. Morgan Department of Radiology, Johns Hopkins University, Baltimore, Maryland; \\ ${ }^{2}$ Nuklearmedizinische Klinik der Technischen Universität München, Munich, Germany; and ${ }^{3}$ Department of Nuclear Medicine, \\ Hannover Medical School, Hannover, Germany
}

Current noninvasive tests for coronary artery disease detect atherosclerosis or regional ischemia. Global myocardial flow reserve is not routinely identified, although it may be an additional marker of disease development and progression. Methods: For the clinical work-up of suspected or known stable coronary artery disease, 275 individuals had undergone rest-dipyridamole ${ }^{82} \mathrm{Rb}$ myocardial perfusion imaging using PET. In addition to clinical measures of regional perfusion and function, an experimentally validated approach to quantify global myocardial flow reserve was used. Follow-up was obtained for $362 \pm 277$ d. Results: Myocardial blood flow and flow reserve showed significant correlation to systemic and cardiac hemodynamics and a weak association with risk factors such as age and history of hyperlipidemia. Flow reserve was expectedly lower in subjects with regional ischemia (1.70 \pm 0.65 vs. $2.31 \pm 0.97$ in those without; $P<0.0001)$, but a wide range was observed in those without regional perfusion abnormalities. We used a composite endpoint of hard and soft events to determine that flow reserve below the median was predictive of adverse outcome in the overall population $(P=0.001)$ and in subjects with normal regional perfusion ( $n=178 ; P=0.036$ ), whereas stress flow was predictive only in the overall population $(P=0.001)$. Ageadjusted multivariate analysis confirmed regional perfusion defects (relative hazard, 2.51; 95\% confidence interval, 1.24-5.10; $P=0.009$ ) and low global flow reserve (relative hazard, 2.93; 95\% confidence interval, 1.30-6.65; $P=0.011$ ) as independent predictors of cardiac events. Conclusion: In clinical cardiac ${ }^{82} \mathrm{Rb}$ PET, globally impaired flow reserve is a relevant marker for predicting short-term cardiovascular events. It may be used for integration with currently established functional and morphologic test results and for guidance of preventive measures, especially in the absence of regional flow-limiting disease.

Received Jul. 30, 2010; revision accepted Jan. 7, 2011

For correspondence or reprints contact: Frank M. Bengel, Department of Nuclear Medicine, Hannover Medical School, Carl-Neuberg-Strasse 1, D-30625 Hannover, Germany.

E-mail: bengel.frank@mh-hannover.de

COPYRIGHT @ 2011 by the Society of Nuclear Medicine, Inc.
Key Words: PET; myocardial flow reserve; microvascular dysfunction; coronary artery disease; myocardial perfusion

J Nucl Med 2011; 52:726-732

DOI: 10.2967/jnumed.110.081828

$\mathbf{I}$ current clinical practice, radionuclide myocardial perfusion imaging is used to detect regional myocardial ischemia and infarction as the clinically overt stages of coronary artery disease (CAD). The obtained diagnostic and prognostic information is used as a gatekeeper to invasive angiography and therapy $(1,2)$. But because of continuous improvements in the pathophysiologic understanding of CAD and in its prevention, there is an increasing emphasis on characterizing disease more accurately. The global flow reserve of the coronary circulation as a whole is thought to be an important component of CAD. It may be impaired not only due to macroscopic flow-limiting disease but also due to coronary microvascular dysfunction (3-5). Yet, currently used clinical imaging protocols do not provide comprehensive measures of global myocardial flow reserve (MFR).

PET is a high-end technique that is increasingly translated from research into broader clinical application $(6,7)$. PET has the potential for absolute quantification of myocardial blood flow (MBF) and MFR as markers of coronary vascular function, as documented in numerous research studies $(3,5)$. But this potential has not yet been exploited for clinical imaging, for which PET images are still mostly analyzed in a qualitative or semiquantitative manner, analogous to the approach established for the widely used SPECT technique (8-10).

In the present study, we sought to explore the full potential of PET in patients referred for clinical myocardial perfusion imaging. We speculated that generating measures of global MFR in combination with the clinical standard analysis of relative regional perfusion and ventricular function would allow for comprehensive characterization of the individual pattern of CAD and provide prognostically relevant information. 


\section{MATERIALS AND METHODS}

\section{Patients}

Consecutive patients $(n=275)$, referred to our PET/CT center for clinical rest-stress myocardial perfusion imaging between January 2007 and March 2009, were included. Exclusion criteria were acute myocardial infarction, unstable angina, clinically overt heart failure, contraindications to dipyridamole stress at the time of imaging, or unavailability of dynamic PET datasets. Clinical characteristics and medication at the time of PET are summarized in Table 1. The study group presented a heterogeneous mix of subjects. Ninety-one subjects had known CAD, for whom the rest-stress myocardial perfusion test was requested to accurately identify disease severity. One hundred sixty-eight subjects represented an overall lower risk group, for which the test was requested to rule out CAD before discharge, after presentation with intermittent chest pain. A small group of 16 patients was referred for noncardiac operation, and of those, only 5 patients had a cancer-related operation. Clinical lab results such as lipid parameters or blood glucose were not consistently available, but a detailed history to define risk factor profile was obtained. Caffeine levels were not measured routinely, but patients were instructed to strictly refrain from any caffeinated substances for $24 \mathrm{~h}$ before testing. Because of its retrospective nature, this project was granted exempt status by the Johns Hopkins Institutional Review Board.

\section{PET Acquisition Protocol}

All imaging was performed on a 64-slice Discovery Rx VCT PET/CT scanner (GE Healthcare). Patients fasted for more than
$4 \mathrm{~h}$. After informed consent, individuals were positioned, and a low-dose (120 kV, 50-100 mA) CT scan for attenuation correction of PET data was acquired during shallow breathing. ${ }^{82} \mathrm{Rb}$-chloride (1,480-1,850 MBq [40-50 mCi]), using a large antecubital intravenous line, was infused, and list-mode 2-dimensional PET images were acquired for $8 \mathrm{~min}$. Then, infusion of dipyridamole was started $(0.56 \mathrm{mg} / \mathrm{kg}, 4 \mathrm{~min})$, and a second dose of ${ }^{82} \mathrm{Rb}$ chloride (1,480-1,850 MBq [40-50 mCi]) was infused 4 min after completion, followed by 8-min list-mode acquisition. Quality control of PET and CT for attenuation correction was performed, and datasets were realigned if necessary (11). List-mode data were resampled to attenuation-corrected static (90-s prescan delay), electrocardiographically gated ( 8 bins), and dynamic images (32 frames: $20 \times 6$ s, $5 \times 12$ s, $4 \times 30$ s, and $3 \times 60$ s).

\section{PET Image Analysis}

Qualitative Perfusion. Static images were reangulated to create short-axis and long-axis slices for visual analysis of regional perfusion. Each of 17 myocardial segments (12) was scored on a scale of 0-4 (normal, mildly, moderately, or severely decreased or absent perfusion) by an experienced reader who was unaware of other data. Summed rest scores, summed stress scores (SSSs), and summed difference scores (SDSs; SSS - summed rest score) were calculated (13).

Left Ventricular Function. Endocardial contours were identified on gated PET to determine left ventricular volumes and left ventricular ejection fraction, using previously validated commercially available software (CardIQ physio; GE Healthcare) (14).

TABLE 1

Clinical Characteristics of Study Group

\begin{tabular}{|c|c|c|}
\hline Variable & Entire study group $(n=275)$ & $\begin{array}{l}\text { Subjects included in outcome } \\
\text { analysis }(n=224)\end{array}$ \\
\hline Age (y) & $57 \pm 12$ & $58 \pm 13$ \\
\hline \multicolumn{3}{|l|}{ Sex } \\
\hline Men & $112(40)$ & $86(38)$ \\
\hline Women & $163(60)$ & $138(62)$ \\
\hline \multicolumn{3}{|l|}{ Race } \\
\hline White & $82(30)$ & $61(27)$ \\
\hline Black & $181(66)$ & $159(71)$ \\
\hline Other & $12(4)$ & $4(2)$ \\
\hline Body mass index $\left(\mathrm{kg} / \mathrm{m}^{2}\right)$ & $32 \pm 9$ & $32 \pm 9$ \\
\hline Known CAD & $91(33)$ & $87(39)$ \\
\hline History of prior myocardial infarction & $30(11)$ & $26(12)$ \\
\hline \multicolumn{3}{|l|}{ Pretest CAD likelihood* } \\
\hline Low & $101(37)$ & $82(37)$ \\
\hline Intermediate & $62(22)$ & $43(19)$ \\
\hline High & $21(8)$ & $12(5)$ \\
\hline History of smoking & $94(34)$ & $83(37)$ \\
\hline History of hypertension & $175(60)$ & $140(63)$ \\
\hline History of hyperlipidemia & $122(44)$ & $101(45)$ \\
\hline History of diabetes mellitus & $90(33)$ & $78(34)$ \\
\hline \multicolumn{3}{|l|}{ Medication at PET } \\
\hline$\beta$-Blocker & $50(18)$ & $32(14)$ \\
\hline $\begin{array}{l}\text { Angiotensin-converting enzyme } \\
\text { inhibitor or angiotensin receptor blocker }\end{array}$ & $58(21)$ & $46(21)$ \\
\hline Statin & $60(22)$ & $44(20)$ \\
\hline
\end{tabular}


Quantification of MBF and MFR. Myocardial activity in the last frame of the dynamic datasets was volumetrically sampled, and polar maps of the left ventricle were generated (15). Segments were applied to the whole series to obtain myocardial time-activity curves. A small region of interest was positioned in the left ventricular cavity to obtain the arterial input function. MBF was then quantified by a retention approach. Myocardial activity concentration between minutes 4 and 8 was normalized to the area under the arterial input function in the first $120 \mathrm{~s}$. The resulting index was corrected for partial volume, spillover, and nonlinear extraction of ${ }^{82} \mathrm{Rb}(16)$. The validity and reproducibility of this approach were recently established for our PET system (17). Because of the relationship of $\mathrm{MBF}$ at rest with the product of heart rate and systolic blood pressure as an index of cardiac work (18), normalization was performed by dividing rest $\mathrm{MBF}$ by the rate-pressure product and multiplying the result by 10,000. Because flow and work are uncoupled during pharmacologic vasodilation, no correction was performed for stress flow. MFR was determined as the ratio of stress $\mathrm{MBF}$ to corrected rest MBF.

\section{Assessment of Outcome}

Patients were followed for occurrence of adverse events by review of electronic medical records at Johns Hopkins Medical Institutions and Social Security Death Index. A composite endpoint was used, which incorporated hard events (cardiac death, myocardial infarction) and soft events (invasive angiography with or without revascularization, rehospitalization for heart failure). Events and cause of death were adjudicated by a consensus of 2 investigators. Subjects with early, test-driven catheterization or revascularization within $90 \mathrm{~d}$ of PET were excluded.

\section{Statistical Analysis}

Data are expressed as mean $\pm \mathrm{SD}$. The Student $t$ test and ANOVA (with post hoc Bonferroni-corrected $t$ test) were used for comparison of normally distributed continuous variables between groups, when appropriate. The Pearson correlation coefficient, with Fisher $r$-to- $z$ transformation for significance, was used to describe relationships between continuous variables.

Patients were grouped according to various PET results, and survival curves were constructed according to Kaplan-Meier. A log-rank analysis was performed to test for significant differences. Then, relative hazards and $95 \%$ confidence intervals were calculated with multivariate Cox proportional hazards regression models. In these models, the relationship of PET-derived variables to outcome was assessed in an age-adjusted manner. Multivariate analyses were performed with stepwise forward regression, with an entry probability for each variable set at 0.05 . Reported $P$ values were 2 -sided; a $P$ value of less than 0.05 was considered to indicate statistical significance.

\section{RESULTS}

\section{Qualitative Regional Perfusion and Left Ventricular Function}

Abnormal stress perfusion (SSS $>4$ ) was observed in 97 of 275 patients $(35 \%)$. Regional ischemia (SDS $>2$ ) was found in 45 of 275 patients $(16 \%)$. Reduced left ventricular ejection fraction $(<45 \%)$ was observed at rest in $60(22 \%)$ and at stress in $55(20 \%)$ of 275 patients.

\section{Global MBF and MFR}

Rest MBF was $0.93 \pm 0.36 \mathrm{~mL} / \mathrm{min} / \mathrm{g}$ and correlated significantly with the rate-pressure product as a measure of cardiac work $(r=0.47 ; P<0.0001)$. After correction for baseline work, rest MBF was $0.94 \pm 0.43 \mathrm{~mL} / \mathrm{min} / \mathrm{g}$. Stress $\mathrm{MBF}$ was $1.97 \pm 0.82 \mathrm{~mL} / \mathrm{min} / \mathrm{g}$, and MFR was $2.21 \pm$ 0.95. MFR correlated with stress MBF $(r=0.46 ; P<$ $0.0001)$ and was inversely correlated with rest MBF before $(r=-0.36 ; P<0.0001)$ and after correction $(r=-0.25$; $P<0.0001$ ) (Fig. 1). Thus, because stress and rest MBF both determined MFR, MFR was used as an integrative marker of global microvascular dysfunction in further analyses.

MFR showed a weak but significant correlation with stress left ventricular ejection fraction $(r=0.13 ; P=$ 0.036) and was expectedly lower in subjects with abnormal SSS $(1.70 \pm 0.65$ vs. $2.31 \pm 0.97$ for normal SSS; $P<$ $0.0001)$ and abnormal SDS (1.73 \pm 0.64 vs. $2.31 \pm 0.98$ for normal SDS; $P<0.0001)$.

MFR was also associated with clinical variables, including an inverse correlation with age $(-0.20 ; P=0.0004)$, lower values in patients with a history of hyperlipidemia $(2.11 \pm 1.05$ vs. $2.27 \pm 0.85$ for those without; $P=0.014)$, and lower values in those with a prior history of CAD (2.01 \pm 0.98 vs. $2.33 \pm 0.91$ for those without; $P=0.0054)$. Of the 30 patients with a prior history of myocardial infarction,
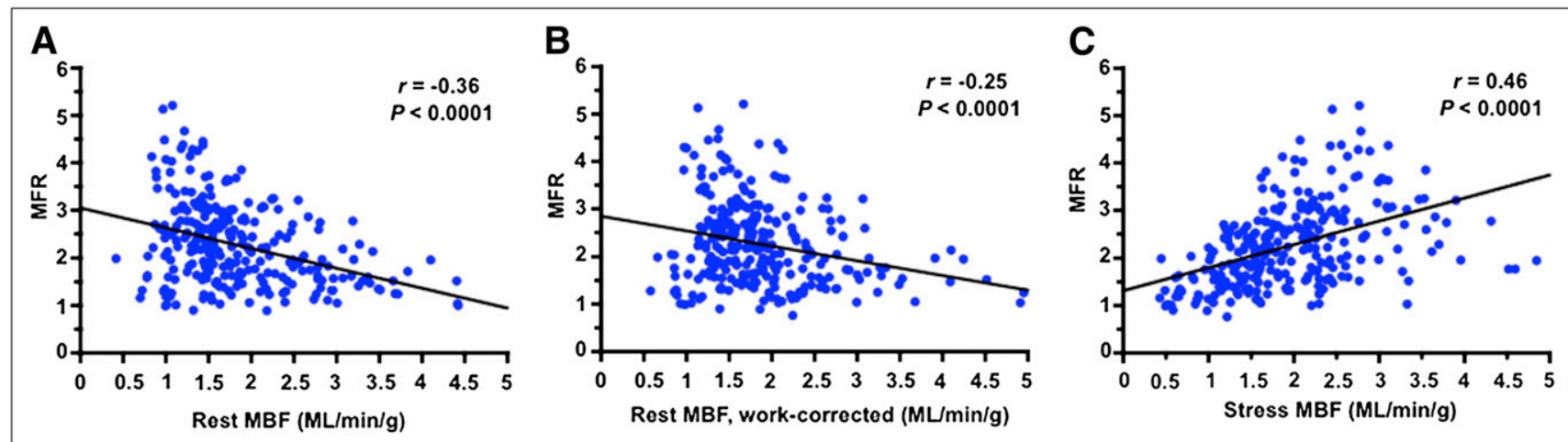

FIGURE 1. Global MFR is determined by dividing absolute MBF during stress by MBF at rest. Shown are regression plots for MFR and rest MBF (A), MFR and rest MBF after correction for baseline cardiac work by normalization for rate-pressure product (B), and MFR and MBF during pharmacologic vasodilation using dipyridamole stress (C). 
22 had abnormal perfusion PET scan results. MFR in this subgroup was $1.50 \pm 0.51$, which was significantly lower than for subjects without myocardial infarction $(2.24 \pm$ $0.89 ; P=0.001)$.

\section{Clinical Outcome}

Twenty-three patients were excluded because of early catheterization or revascularization after PET. Twenty-eight were lost to follow-up. These patients did not show any difference in their PET results when compared with subjects who were included in outcomes analysis (SSS, $1 \pm 3$ vs. $3 \pm 7$ in included subjects, $P=0.12$; MFR, $2.4 \pm 0.9$ vs. $2.2 \pm 0.9, P=0.26$; and left ventricular ejection fraction, $60 \pm 12$ vs. $56 \pm 14, P=0.09$ ).

In the remaining 224 patients, mean follow-up time was $362 \pm 277 \mathrm{~d}$ (median, $426 \mathrm{~d}$; range, 4-924 d). During follow-up, 33 patients $(15 \%)$ had a cardiac event (cardiac death, $n=9$; myocardial infarction, $n=7$; late revascularization, $n=2$; late cardiac catheterization without intervention, $n=9$; and readmission for heart failure, $n=6$ ). Time to event was $196 \pm 162 \mathrm{~d}$ (median, $182 \mathrm{~d}$; range, 9$950 \mathrm{~d})$. Three patients died because of noncardiac disease ( 2 deaths related to cancer and 1 due to terminal lung disease). Data for these patients were censored at the time of death. Among the 9 patients who died of cardiac disease, 8 had known CAD at the time of PET.

Kaplan-Meier analysis confirmed a significantly better outcome for patients with normal than for those with abnormal regional perfusion ( $P<0.0001$; Fig. 2A). A significantly better event-free survival was also observed for stress MBF and MFR above the median than for stress MBF and MFR below $(P=0.001$ for stress MBF and MFR; Figs. 2B and 2C). Age-adjusted multivariate Cox proportional hazards analysis showed that SSS and MFR were independent predictors of an unfavorable event (Table 2 ). When analysis was restricted to 178 patients without regional perfusion abnormalities, higher MFR was still associated with better outcome $(P=0.026$; Fig. 3B), whereas stress $\mathrm{MBF}$ was no longer predictive of adverse outcome ( $P=0.21$; Fig. $3 \mathrm{~A})$.

From the 8 patients with previous CAD who experienced cardiac death, only 2 patients had a history of myocardial infarction, and 4 had reduced ejection fraction. When Kaplan-Meyer and Cox proportional hazards analyses were repeated in all patients except those with myocardial infarction, results remained unchanged $(P=0.001$ for subjects with higher vs. lower flow reserve at Kaplan-Meier; $P=$ 0.005 for lower flow reserve and $P=0.006$ for SSS as the 2 independent predictors of cardiac events in Cox proportional hazards analysis).

\section{DISCUSSION}

Our results confirm the usefulness of MFR quantification for the noninvasive work-up of CAD. Reduced global flow reserve was indicative of short-term adverse events in the entire population undergoing clinical myocardial perfusion
PET, but also in a subgroup that showed no evidence of significant disease by other results of the comprehensive test.

In the absence of flow-limiting epicardial coronary artery stenoses and myocardial disease, impaired flow reserve is thought to reflect microvascular dysfunction as a consequence of atherosclerotic risk factors and thus an early stage of disease. This finding is supported by several reports of PET-defined microvascular disease in well-selected subjects with a single risk factor such as smoking, hyperlipidemia, and insulin resistance (18-22). Other studies showed that impaired flow dynamics in such subjects can be improved by various interventions (19,23-25), suggesting that it is a reversible condition. Most of our patients (i.e., those who did not show evidence of regional ischemia on qualitative PET images) seem to fall into this category, and our study shows that reduced flow reserve is predictive of an unfavorable outcome. In contrast to these studies, however, strong correlations of flow reserve with one or more risk factors were not observed in our study. This difference may be explained by the heterogeneous mix of individuals with varying risk profiles, different degrees of preexisting atherosclerosis, and different concomitant medications, which is commonly encountered in the clinical setting of CAD work-up. The lower extraction of ${ }^{82} \mathrm{Rb}$ at higher flow, when compared with alternative PET tracers, may be another factor that attenuates such correlations. And finally, microvascular angina (syndrome X) may have been another contributor to low flow reserve in this group. No component of the PET test other than global flow quantification would have been able to identify risk in subjects without regional perfusion abnormalities, suggesting an added clinical value. It is tempting to speculate that the addition of flow quantification to standard noninvasive testing may refine the detection of early disease stages and help to improve preventive measures to reverse risk. Our study provides a foundation for future prospective trials to test this hypothesis.

Although impaired flow reserve is a marker of microvascular dysfunction in the absence of flow-limiting epicardial disease, the situation is different and more complex in the presence of obstructive, flow-limiting CAD. In this setting, global flow reserve reflects a composite marker of the degree of flow limitation by macroscopic stenosis and the degree of microvascular dysfunction. These can be distinguished only if information about coronary anatomy is available. Unfortunately, because of the retrospective nature of our study, because invasive angiography is not indicated in cases with normal relative regional perfusion, and because combined assessment by CT angiography and perfusion imaging is not yet a clinical standard, morphologic studies were not consistently available in our study population. Other studies have shown that flow reserve declines with increasing degree of stenosis (26), but microvascular dysfunction may determine the threshold of ischemia in the territory of the stenotic artery (27). Furthermore, it has been shown in single-vessel disease that flow reserve can be abnormal in remote regions subtended by normal 


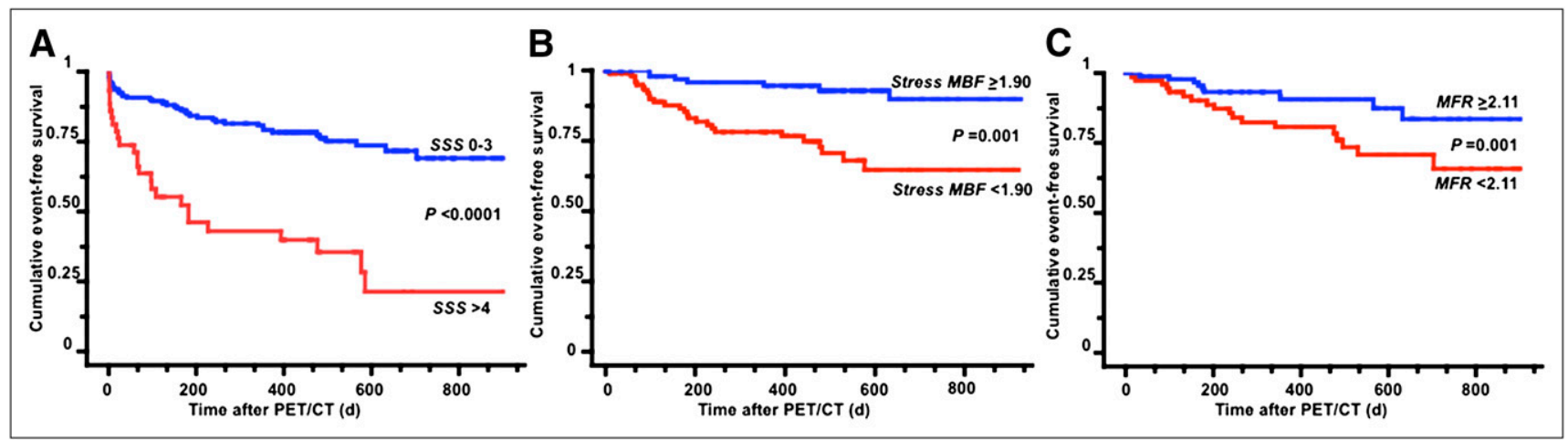

FIGURE 2. Prognostic value of myocardial perfusion, myocardial stress flow, and MFR for entire population. Survival curves according to Kaplan-Meier for abnormal vs. normal relative regional perfusion in all subjects $(n=225)(A)$, lower vs. upper half of quantitative stress myocardial flow (stress MBF) (B), and MFR as measure of microvascular function in all subjects (C).

macroscopic arteries (28). In the extreme setting of balanced, flow-limiting triple-vessel disease, global flow reserve may be severely reduced in the absence of regional flow heterogeneity (29). This would not allow for conclusions about the microcirculation but would still identify a state of high risk. Given their clinical profile, which mostly indicated low risk, this situation is unlikely among our subjects with normal regional perfusion. But in the absence of angiographic results, balanced ischemia cannot be ruled out completely as a contributor to the association between low flow reserve and adverse outcome in subjects with absence of regional perfusion heterogeneity. Our results nevertheless suggest a prognostic value of global flow reserve in subjects referred for work-up of CAD, for which it may be another marker of risk in addition to the well-known prognostic value of regional perfusion defect size, which has been well established not only for SPECT (30) but also for ${ }^{82} \mathrm{Rb}$ PET $(9,31,32)$.

Several prior studies have reported a prognostic value of PET-derived flow reserve in cardiomyopathies $(33,34)$ and in subjects with and without known CAD (35-37). However, these studies had in common only that the cyclotronproduced tracers ${ }^{13} \mathrm{~N}$-ammonia or ${ }^{15} \mathrm{O}$-water was used. Although those tracers are considered a gold standard, they will remain limited to few academic institutions, and they may not reach clinical acceptance because they cannot be broadly distributed. Our study is innovative in that it reports about the prognostic value of flow reserve in the general clinical setting of perfusion PET with ${ }^{82} \mathrm{Rb}$, which is a widely commercially available generator product and increasingly used for noninvasive CAD assessment (7). Current systems routinely feature list-mode PET acquisition, which synchronously enables the sampling of dynamic datasets that are necessary for flow quantification, along with static data for standard regional perfusion analysis and electrocardiographically gated datasets for standard functional analysis (6). ${ }^{82} \mathrm{Rb}$ has potential disadvantages for absolute flow quantification because of physical properties and nonlinear extraction fraction in the high-flow range. But several algorithms for quantification have been validated and seem to work in the setting of CAD work-up, in which high flow is uncommon $(16,17,38,39)$. Although software for flow quantification is not yet commercially available, our results support a move forward with the commercialization of such products so that the assessment of microvascular function can be more broadly implemented into clinical noninvasive imaging practice.

Another interesting aspect of our study is that global stress flow alone was predictive of outcome in our entire study group at univariate analysis. However, flow reserve, which

TABLE 2

Results of Multivariate Cox Regression Analyses Assessing Relationship Between PET Variables and Outcome, Adjusted for Age

\begin{tabular}{lccc}
\hline \multicolumn{1}{c}{ Variable } & Relative hazard & $95 \%$ confidence interval & $P$ \\
\hline MFR $<2.11$ & 2.93 & $1.30 \%-6.65 \%$ & 0.009 \\
Abnormal SSS $>4$ & 2.51 & $1.24 \%-5.10 \%$ & 0.011 \\
Stress myocardial flow $<1.90 \mathrm{~mL} / \mathrm{min} / \mathrm{g}$ & $-^{*}$ & & 0.09 \\
\hline Stress left ventricular ejection fraction $<45 \%$ & $-^{*}$ & & 0.10
\end{tabular}

*Variables that were removed from final model.

All models were adjusted for age as stratification factor. Patients were divided into 3 equal groups based on age. Cutoffs for groups were $24-51,52-62$, and $63-88$. $P$ value denotes level of significance that led to exclusion of variables. 


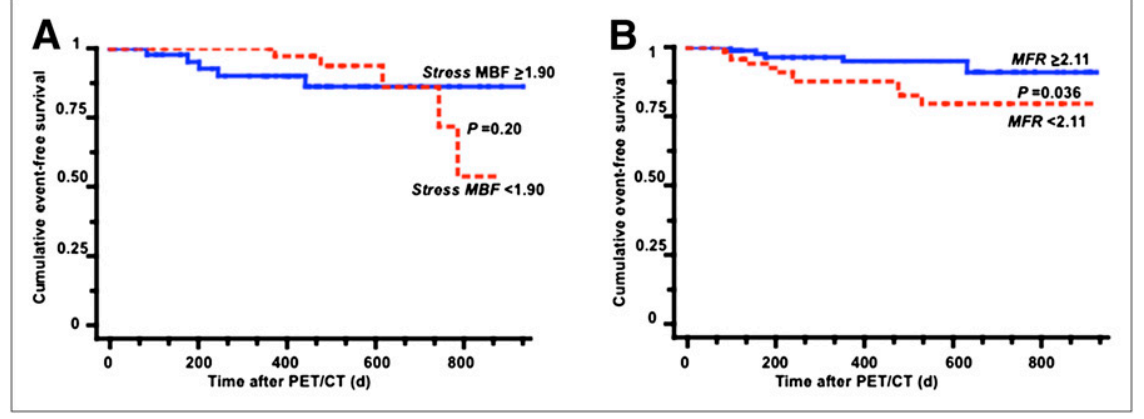

FIGURE 3. Prognostic value of MFR and stress MBF for patients with normal regional perfusion, as defined by SSS below 4 ( $n=$ 178). Survival curves according to KaplanMeier for lower vs. upper half of quantitative stress myocardial flow (stress MBF) (A) and MFR (B). In normal-perfusion patients, MFR was still predictive for adverse outcome whereas stress MBF did not show significant predictability for events. integrates rest and stress flow, was superior at multivariate analysis and retained its prognostic value in the subgroup without regional perfusion defects, whereas stress flow alone was no longer predictive on univariate analysis (and therefore not used for a multivariate analysis). These data suggest that both rest and stress measurements are necessary, especially in the absence of obstructive epicardial disease, to obtain the most accurate prognostic information.

It should be recognized that our study has several limitations. First, because of the small sample size and short observation period, events are limited and the statistical power for identification of a truly incremental prognostic value is limited. For the same reason, and to avoid overfitting (40), we did not perform more complex multivariate outcome analyses, adjustments for multiple risk factors other than age, and analyses of interaction terms among imaging variables and risk factors.

Second, the retrospective nature and the use of a composite endpoint are further limitations. Because of the low frequency of death and myocardial infarction in our relatively healthy population, we included other events such as revascularization and hospital readmission to increase power. Flow reserve measurements were conducted offline and were not available to clinical physicians. Clinical decision making was thus not influenced by flow quantification. The assumption of risk homogeneity among the included endpoints in our study, however, may not hold true, because soft events such as angiography or readmission were used alongside hard events such as death or myocardial infarction (40). Nevertheless, all included endpoints represent significant events that resulted either in increased costs of care or reduced quality of life.

Third, our study is a single-center report, obtained from results of an unselected consecutive group of patients, as reflects local clinical practice at our institution. Clinical characteristics suggest a relatively low risk. The percentage of studies with abnormal regional perfusion is, at $35 \%$, not high but within the range of what has been described by others (31). Only a fraction of subjects underwent early angiography after a positive PET study. Multiple factors may have played a role, such as socioeconomic issues in the local patient population (lack of insurance coverage, lack of consent to recommended procedure, expected lack of compliance with antiplatelet therapy after intervention) and an increasing awareness of referring physicians that conservative therapy may be beneficial even in mildly to moderately abnormal perfusion studies. This low rate of early angiography may even be beneficial in that it reduces bias-because of the low rate of early intervention, fewer subjects with positive scan results had to be removed from follow-up.

Fourth, because we intentionally aimed at analyzing the value of quantitative flow reserve in an unselected group of patients referred for myocardial perfusion PET, patients with a history of myocardial infarction were not excluded. Prior myocardial infarction is expected to contribute to reduced flow reserve. Results of our study confirm an independent prognostic value in all subjects and after exclusion of subjects with prior infarctions, suggesting that flow reserve is an integrated risk marker independent of prior cardiac events.

And finally, the retrospective nature, along with the profile of our patients, resulted in a relatively large fraction of subjects who were lost to follow-up. Although an effect on the results of the study cannot be completely excluded, the drop-out group did not differ from other subjects with regard to their scan results.

It is important to emphasize that this initial, preliminary report should be seen as a stimulus to include flow quantification in future large-scale prospective outcome trials. Such trials may then provide the evidence needed for a broad implementation of quantitative flow measurements in clinical perfusion imaging practice.

\section{CONCLUSION}

Findings of our limited-size, retrospective analysis support the notion that implementation of absolute quantification of MFR may augment clinical myocardial perfusion imaging, by improving the identification of individuals at higher risk of adverse events within the first 1-2 y after the test. PET with the broadly available perfusion tracer ${ }^{82} \mathrm{Rb}$ may be used for this purpose and may be implemented into efforts at early detection and reversal of CAD in the future.

\section{DISCLOSURE STATEMENT}

The costs of publication of this article were defrayed in part by the payment of page charges. Therefore, and solely 
to indicate this fact, this article is hereby marked "advertisement" in accordance with 18 USC section 1734.

\section{ACKNOWLEDGMENTS}

This study was supported in part by a Wagner-Torizuka Fellowship grant from the Society of Nuclear Medicine, GE Healthcare, Bracco Diagnostics, and Lantheus Medical Imaging.

\section{REFERENCES}

1. Klocke FJ, Baird MG, Lorell BH, et al. ACC/AHA/ASNC guidelines for the clinical use of cardiac radionuclide imaging-executive summary: a report of the American College of Cardiology/American Heart Association Task Force on Practice Guidelines (ACC/AHA/ASNC Committee to Revise the 1995 Guidelines for the Clinical Use of Cardiac Radionuclide Imaging). Circulation. 2003;108:1404-1418.

2. Marcassa C, Bax JJ, Bengel F, et al. Clinical value, cost-effectiveness, and safety of myocardial perfusion scintigraphy: a position statement. Eur Heart J. 2008;29:557-563.

3. Camici PG, Crea F. Coronary microvascular dysfunction. N Engl J Med. 2007; 356:830-840.

4. Halcox JP, Schenke WH, Zalos G, et al. Prognostic value of coronary vascular endothelial dysfunction. Circulation. 2002;106:653-658.

5. Schelbert HR. Quantification of myocardial blood flow: what is the clinical role? Cardiol Clin. 2009;27:277-289.

6. Bengel FM, Higuchi T, Javadi MS, Lautamaki R. Cardiac positron emission tomography. J Am Coll Cardiol. 2009;54:1-15.

7. Di Carli MF, Hachamovitch R. New technology for noninvasive evaluation of coronary artery disease. Circulation. 2007;115:1464-1480.

8. Bateman TM, Heller GV, McGhie AI, et al. Diagnostic accuracy of rest/stress ECG-gated Rb-82 myocardial perfusion PET: comparison with ECG-gated Tc99m sestamibi SPECT. J Nucl Cardiol. 2006;13:24-33.

9. Lertsburapa K, Ahlberg AW, Bateman TM, et al. Independent and incremental prognostic value of left ventricular ejection fraction determined by stress gated rubidium 82 PET imaging in patients with known or suspected coronary artery disease. J Nucl Cardiol. 2008;15:745-753.

10. Merhige ME, Breen WJ, Shelton V, Houston T, D'Arcy BJ, Perna AF. Impact of myocardial perfusion imaging with PET and ${ }^{82} \mathrm{Rb}$ on downstream invasive procedure utilization, costs, and outcomes in coronary disease management. $\mathrm{J} \mathrm{Nucl}$ Med. 2007;48:1069-1076.

11. Lautamaki R, Brown TL, Merrill J, Bengel FM. CT-based attenuation correction in ${ }^{82} \mathrm{Rb}$-myocardial perfusion PET-CT: incidence of misalignment and effect on regional tracer distribution. Eur J Nucl Med Mol Imaging. 2008;35:305-310.

12. Cerqueira MD, Weissman NJ, Dilsizian V, et al. Standardized myocardial segmentation and nomenclature for tomographic imaging of the heart: a statement for healthcare professionals from the Cardiac Imaging Committee of the Council on Clinical Cardiology of the American Heart Association. Circulation. 2002;105:539-542.

13. Hansen CL, Goldstein RA, Akinboboye OO, et al. Myocardial perfusion and function: single photon emission computed tomography. J Nucl Cardiol. 2007; 14:e39-e60.

14. Chander A, Brenner M, Lautamaki R, Voicu C, Merrill J, Bengel FM. Comparison of measures of left ventricular function from electrocardiographically gated ${ }^{82} \mathrm{Rb}$ PET with contrast-enhanced CT ventriculography: a hybrid PET/CT analysis. J Nucl Med. 2008;49:1643-1650.

15. Nekolla SG, Miethaner C, Nguyen N, Ziegler SI, Schwaiger M. Reproducibility of polar map generation and assessment of defect severity and extent assessment in myocardial perfusion imaging using positron emission tomography. Eur J Nucl Med. 1998;25:1313-1321.

16. Yoshida K, Mullani N, Gould KL. Coronary flow and flow reserve by PET simplified for clinical applications using rubidium-82 or nitrogen-13-ammonia. J Nucl Med. 1996;37:1701-1712.

17. Lautamaki R, George RT, Kitagawa K, et al. Rubidium-82 PET-CT for quantitative assessment of myocardial blood flow: validation in a canine model of coronary artery stenosis. Eur J Nucl Med Mol Imaging. 2009;36:576-586.

18. Czernin J, Muller P, Chan S, et al. Influence of age and hemodynamics on myocardial blood flow and flow reserve. Circulation. 1993;88:62-69.
19. Czernin J, Barnard RJ, Sun KT, et al. Effect of short-term cardiovascular conditioning and low-fat diet on myocardial blood flow and flow reserve. Circulation. 1995;92:197-204.

20. Dayanikli F, Grambow D, Muzik O, Mosca L, Rubenfire M, Schwaiger M. Early detection of abnormal coronary flow reserve in asymptomatic men at high risk for coronary artery disease using positron emission tomography. Circulation. 1994;90:808-817.

21. Kaufmann PA, Gnecchi-Ruscone T, Schafers KP, Luscher TF, Camici PG. Low density lipoprotein cholesterol and coronary microvascular dysfunction in hypercholesterolemia. J Am Coll Cardiol. 2000;36:103-109.

22. Pitkanen OP, Nuutila P, Raitakari OT, et al. Coronary flow reserve is reduced in young men with IDDM. Diabetes. 1998;47:248-254.

23. Gould KL, Ornish D, Scherwitz L, et al. Changes in myocardial perfusion abnormalities by positron emission tomography after long-term, intense risk factor modification. JAMA. 1995;274:894-901.

24. Kaufmann PA, Gnecchi-Ruscone T, di Terlizzi M, Schafers KP, Luscher TF, Camici PG. Coronary heart disease in smokers: vitamin C restores coronary microcirculatory function. Circulation. 2000;102:1233-1238.

25. Higuchi T, Abletshauser C, Nekolla SG, Schwaiger M, Bengel FM. Effect of the angiotensin receptor blocker Valsartan on coronary microvascular flow reserve in moderately hypertensive patients with stable coronary artery disease. Microcirculation. 2007;14:805-812.

26. Uren NG, Melin JA, De Bruyne B, Wijns W, Baudhuin T, Camici PG. Relation between myocardial blood flow and the severity of coronary-artery stenosis. $N$ Engl J Med. 1994;330:1782-1788.

27. Pupita G, Maseri A, Kaski JC, et al. Myocardial ischemia caused by distal coronary-artery constriction in stable angina pectoris. N Engl J Med. 1990; 323:514-520.

28. Sambuceti G, Marzullo P, Giorgetti A, et al. Global alteration in perfusion response to increasing oxygen consumption in patients with single-vessel coronary artery disease. Circulation. 1994;90:1696-1705.

29. Parkash R, deKemp RA, Ruddy TD, et al. Potential utility of rubidium 82 PET quantification in patients with 3-vessel coronary artery disease. J Nucl Cardiol. 2004;11:440-449.

30. Hachamovitch R, Berman DS, Shaw LJ, et al. Incremental prognostic value of myocardial perfusion single photon emission computed tomography for the prediction of cardiac death: differential stratification for risk of cardiac death and myocardial infarction. Circulation. 1998;97:535-543.

31. Schenker MP, Dorbala S, Hong EC, et al. Interrelation of coronary calcification, myocardial ischemia, and outcomes in patients with intermediate likelihood of coronary artery disease: a combined positron emission tomography/computed tomography study. Circulation. 2008;117:1693-1700.

32. Yoshinaga K, Chow BJ, Williams $\mathrm{K}$, et al. What is the prognostic value of myocardial perfusion imaging using rubidium- 82 positron emission tomography? J Am Coll Cardiol. 2006;48:1029-1039.

33. Cecchi F, Olivotto I, Gistri R, Lorenzoni R, Chiriatti G, Camici PG. Coronary microvascular dysfunction and prognosis in hypertrophic cardiomyopathy. $N$ Engl J Med. 2003;349:1027-1035.

34. Neglia D, Michelassi C, Trivieri MG, et al. Prognostic role of myocardial blood flow impairment in idiopathic left ventricular dysfunction. Circulation. 2002; 105:186-193.

35. Herzog BA, Husmann L, Valenta I, et al. Long-term prognostic value of ${ }^{13} \mathrm{~N}-$ ammonia myocardial perfusion positron emission tomography added value of coronary flow reserve. J Am Coll Cardiol. 2009;54:150-156.

36. Schindler TH, Nitzsche EU, Schelbert HR, et al. Positron emission tomographymeasured abnormal responses of myocardial blood flow to sympathetic stimulation are associated with the risk of developing cardiovascular events. J Am Coll Cardiol. 2005;45:1505-1512.

37. Tio RA, Dabeshlim A, Siebelink HM, et al. Comparison between the prognostic value of left ventricular function and myocardial perfusion reserve in patients with ischemic heart disease. J Nucl Med. 2009;50:214-219.

38. El Fakhri G, Kardan A, Sitek A, et al. Reproducibility and accuracy of quantitative myocardial blood flow assessment with ${ }^{82} \mathrm{Rb}$ PET: comparison with ${ }^{13} \mathrm{~N}$ ammonia PET. J Nucl Med. 2009;50:1062-1071.

39. Lortie M, Beanlands RS, Yoshinaga K, Klein R, Dasilva JN, DeKemp RA. Quantification of myocardial blood flow with ${ }^{82} \mathrm{Rb}$ dynamic PET imaging. Eur J Nucl Med Mol Imaging. 2007;34:1765-1774.

40. Hachamovitch R, Di Carli MF. Methods and limitations of assessing new noninvasive tests: Part II: outcomes-based validation and reliability assessment of noninvasive testing. Circulation. 2008;117:2793-2801. 